\title{
LES TUMEURS PAROTIDIENNES DE L'ENFANT A PROPOS DE 13 CAS
}

\author{
M. BELLAKHDHAR, I. ZEGLAOUI, M. ABDELKÉFI, R. FDHILA, \\ R. MANI, M. BEN ALI, M. BELCADHI, K. BOUZOUITA . \\ SERVICE D'ORL ET DE CHIRURGIE CERVICOFACIALE. \\ CHU FARHAT HACHED. SOUSSE.
}

\begin{abstract}
Introduction : Les tumeurs parotidiennes de l'enfant sont rares. Plusieurs types histologiques peuvent être rencontrés et rappellent souvent ceux de l'adulte.

But : Analyser les particularités épidémiologiques, cliniques et thérapeutiques des tumeurs parotidiennes chez l'enfant. Matériel et méthodes : Il s'agit d'une étude rétrospective, portant sur 13 observations de tumeurs parotidiennes de l'enfant colligées sur une période de 23 ans de Janvier 1985 à Décembre 2007.

Résultats : Nos malades se répartissaient en 8 filles et 5 garçons. L'âge moyen était de 11 ans.

Notre série avait comporté 12 cas $(92,3 \%)$ de tumeurs bénignes et un cas $(7,7)$ de tumeur maligne.

Tous nos malades ont eu un traitement chirurgical.

L'examen histologique définitif a conclu à un adénome pléomorphe dans 5 cas, un oncocytome dans un cas. Deux malades étaient porteurs d'une tumeur vasculaire, un malade d'une tumeur nerveuse, deux de kystes lymphoépithéliaux, un malade d'un kyste hydatique et un patient d'un carcinome mucoépidermoïde.

L'évolution a été marquée par l'absence de récidive tumorale avec un recul variant entre 8 mois et 6 ans pour tous nos malades.

Conclusion : Bien que les tuméfactions parotidiennes soient rares chez l'enfant, elles représentent une grande variété de diagnostics histologiques y compris des tumeurs malignes.
\end{abstract}

Mots-clés : Glande parotide, Enfant, Tumeurs, Parotidectomie, Radiothérapie.

\begin{abstract}
Introduction : Parotid masses in children are uncommon and consist of various lesions. Some histological types remind those of the adult.

Purpose : Analyze of the epidemiological, clinical and therapeutic particularities of pediatric parotid tumors.

Patients : Thirteen cases of pediatric parotid tumors treated over a 23 year period between January 1985 - December 2007 in the ENT department of Farhat Hached hospital.

Results : Out of a series of 13 cases there were 8 girls and 5 boys aged between 19 months and 15 years with a mean age of 11 years. Twelve cases $(92,3 \%)$ had benign tumors and one patient $(7,7 \%)$ had a malignant tumor. All our patients had a surgical treatment. The histologic diagnosis was: pleomorphic adenoma in five cases, oncoytoma in one case, hemangioma in two cases, schwanoma in one case, lymphoepithelial cyst in two cases. One patient had an hydatid cyst and one had a mucoepidermoid carcinoma.

All patients were free of recurrent disease at the time of last follow-up (range 8 months - 15 years, mean 6 years).

Conclusion : Although parotid tumors are uncommon in children, they can represent a variety of pathological diagnoses, including malignancy.
\end{abstract}

Keywords : Parotid gland, Children, Tumors, Parotidectomy, Radiotherapy.

\section{INTRODUCTION}

Les tumeurs parotidiennes de l'enfant sont rares. Elles représentent 3 à $5 \%$ de l'ensemble des tumeurs parotidiennes tout âge confondu (1).

A travers 13 observations de tumeurs parotidiennes de l'enfant, colligées au service d'ORL et de CCF de l'hôpital Farhat Hached de Sousse, nous nous proposons d'analyser les différents aspects épidémiologiques, cliniques et thérapeutiques de ces tumeurs parotidiennes chez l'enfant.

\section{MATÉRIEL ET MÉTHODES}

II s'agit d'une étude rétrospective portant sur 13 observations de tumeurs parotidiennes de l'enfant colligées sur une période de 23 ans de Janvier 1985 à Décembre 2007.

Durant cette même période, 223 tumeurs parotidiennes ont été opérées, tout âge confondu.

Nos malades se répartissaient en 8 filles et 5 garçons.

L'âge moyen était de 11 ans avec des extrêmes de 19 mois à 15 ans. (Tableau I)

Le délai de consultation était de 1 à 3 ans avec un délai 


\section{A) Les tumeurs bénignes :}

Les tumeurs bénignes sont les tumeurs parotidiennes les plus fréquentes chez l'enfant. Dans la série d'Orvidas (1), $83,9 \%$ des tumeurs parotidiennes étaient bénignes. Ce taux était de $75 \%$ pour Ethunandan (2). Dans notre série, le taux de tumeurs bénignes était de 92,3\%.

\section{1) L'adénome pléomorphe :}

II représente la tumeur bénigne la plus fréquente de la glande parotide chez l'enfant $(1,2)$.

Orvidas (1) dans une série de 118 tumeurs parotidiennes de l'enfant a relevé ce type histologique dans 27 cas (22,9\%).

Pour la plupart des auteurs, il n'y a pas de prédominance de sexe $(2,3)$. Cependant d'autres retrouvent une prédominance féminine $(4,5)$.

Sur le plan clinique, l'adénome pléomorphe se présente comme une masse bien limitée, ferme et indolore de la parotide. Le signe de Nélaton n'est pas toujours présent.

Dans notre série nous avons colligé 5 cas d'adénomes pléomorphes. II s'agissait de 4 filles et d'un garçon dont l'âge moyen était de 12 ans. Ils avaient tous consulté pour une tuméfaction de la loge parotidienne. A l'examen, ils avaient tous une masse ferme indolore de la loge parotidienne et le signe de Nélaton a été trouvé chez 2 malades. Non traité, l'adénome pléomorphe peut dégénérer dans 2 à $7 \%$ des cas chez l'adulte (6). Chez l'enfant, cette transformation maligne est exceptionnelle $(3,4,5)$.

Sur le plan thérapeutique, la divergence d'attitude chirurgicale ne se pose que pour les adénomes pléomorphes du lobe superficiel et du pôle inférieur de la glande. Les localisations du lobe profond nécessitent une parotidectomie totale conservatrice du nerf facial (3).

Les arguments qui plaident pour la parotidectomie totale sont essentiellement la plurifocalité de la tumeur, les risques de reprise chirurgicale pour le nerf facial et le potentiel de dégénérescence $(4,5)$.

D'autres pensent que tant que la masse tumorale reste exofaciale, une parotidectomie partielle suffit à en assurer l'exérèse, car, même l'ablation du lobe profond ne pourrait enlever de façon certaine des nodules microscopiques au contact des filets nerveux (3).

Dans la série de Rodriguez (5), 2 malades (18\%) ont présenté une récidive, l'un après une chirurgie partielle et l'autre après une parotidectomie totale. Pour Orvidas (1), 4 malades $(15 \%)$ ont présenté une récidive dont 3 après parotidectomie partielle et 1 après parotidectomie exofaciale.

Tous nos malades ont eu une parotidectomie totale conservatrice du nerf facial. L'évolution était bonne, sans récidive, avec un recul allant de 8 mois à 6 ans.

2) L'oncocytome :

L'oncocytome est une tumeur bénigne, caractérisée par la prolifération de cellules oncocytaires.

II est rare, moins de $1 \%$ de toutes les tumeurs des glandes salivaires, et survient essentiellement au niveau de la parotide (7).

Trois types de lésions oncocytaires sont décrits. Une forme disséminée et non encapsulée, une forme nodulaire multifocale et une forme unique encapsulée.

II n'existe pas de transformation maligne de ces lésions (8). La tumeur se présente souvent comme une masse solide, indolore, multilobulée de la parotide.

Le traitement est chirurgical. Une parotidectomie totale n'est pas toujours indiquée sauf dans les cas où l'oncocytome est multifocal (9).

Sakai (7) a rapporté un cas d'oncocytome du lobe profond de la parotide ayant nécessité une parotidectomie totale. L'évolution était bonne avec un recul de 2 ans.

Dans notre série, nous avons colligé un cas d'oncocytome chez un garçon de 9 ans qui avait consulté pour une masse sous-auriculaire gauche ferme bosselée et à surface régulière. II a eu une parotidectomie exofaciale. L'évolution était bonne sans récidive avec un recul de 9 ans.

\section{3) Les tumeurs vasculaires :}

Les tumeurs vasculaires sont dominées par les hémangiomes qui se voient surtout chez le nouveau-né et l'enfant au cours des premières années de la vie (10).

Ces hémangiomes sont généralement de type capillaire, pouvant avec le temps, subir une régression spontanée. Ceci les différencie de ceux de l'adulte qui sont de type caverneux sans aucune tendance à la régression, nécessitant toujours une exérèse chirurgicale.

Dans la série d'Orvidas (1), parmi les 12 cas d'hémangiomes, $83 \%$ ont été traités chirurgicalement et une surveillance clinique a été préconisée dans $17 \%$ des cas avec une régression spontanée des lésions.

Du fait des difficultés opératoires souvent rencontrées et de la possibilité d'une régression tumorale spontanée, une simple surveillance clinique de ces tumeurs est indiquée. Une exérèse chirurgicale ne doit être réalisée qu'en cas d'aggravation clinique (augmentation du volume, compression du nerf facial) ou de doute diagnostique $(10,11)$.

D'autres traitements peuvent être indiqués : les corticoïdes qui ont un effet anti-anabolisant sur le tissu vasculaire embryonnaire, ou les injections sclérosantes qui diminuent le volume tumoral en provoquant la fibrose (10).

Dans la série de 21 enfants porteurs d'hémangiomes parotidiens rapportée par Buckmiller (11), tous les malades ont eu une injection de stéroïdes ; l'évolution était marquée par la régression totale des lésions dans $52 \%$ des cas, $33,3 \%$ ont eu une régression partielle. Une chirurgie a été indiquée dans $7,3 \%$ des cas devant l'absence de régression ou l'augmentation de la taille de la lésion sous traitement. Pour nos deux malades, l'un a eu une parotidectomie totale conservatrice du nerf facial, et l'autre une tumorectomie devant une formation tumorale de siège périphérique. L'examen anatomopathologique a conclu à un angiome capillaire dans le premier cas et à un hémangiome caverneux dans le deuxième cas. 


\section{4) Les tumeurs nerveuses :}

Les tumeurs nerveuses bénignes intra-parotidiennes sont rares et représentées par les shwannomes et les neurofibromes (12).

Les shwannomes du nerf facial sont rares. Depuis le premier cas décrit par Schmidt en 1931 (13), 467 cas de schwannomes du nerf facial ont été rapportés dans la littérature. La portion intra parotidienne du nerf facial est rarement touchée (13).

Tous les âges sont concernés sans prédominance de sexe. Les symptômes majeurs associés au schwannome sont les spasmes hémifaciaux et les paralysies faciales qui sont en rapport avec une compression ou une infiltration du nerf facial. Ces symptômes sont absents dans $50 \%$ des localisations intra-parotidiennes.

Dans notre série, le diagnostic de schwannome a été posé chez une fillette de 10 ans qui avait consulté pour une tuméfaction sous-lobulaire droite isolée sans paralysie faciale ni spasmes associés.

Aucun examen paraclinique n'a de valeur diagnostique, le diagnostic positif est histologique.

Le traitement est chirurgical. Cependant, et du fait de l'évolution lente de la tumeur, une surveillance clinique peut être envisagée en l'absence de paralysie faciale associée (14). Dans la série de Robert (14), comportant 29 malades porteurs de shwannomes intra-parotidiens, 26 ont eu un traitement chirurgical, avec une résection tumorale dans 24 cas et une biopsie tumorale avec décompression du nerf facial dans 2 cas.

Pour notre malade l'évolution après traitement chirurjical était bonne avec un recul de 7 ans.

5) Les tumeurs kystiques :

Les kystes de la parotide sont rares et représentent $5 \%$ de toutes les tumeurs des glandes salivaires (15).

* Les kystes lymphoépithéliaux :

Parmi les kystes malformatifs, le kyste lymphoépithélial est le plus fréquent.

Ils sont considérés comme une très rare manifestation du syndrome de Sjögren et sont aussi une manifestation ORL du virus d'immunodépression humaine (HIV) notamment lorsque les lésions sont multiples et bilatérales. James (16), dans une série de 60 malades porteurs de kystes lymphoépithéliaux, a retrouvé une sérologie positive pour le HIV chez 38 malades.

Dans notre série, nous rapportons deux cas de kystes lymphoépithéliaux.

La sérologie HIV, réalisée chez une malade âgée de 14 ans porteuse de multiples lésions lymphoépithéliales, était négative.

Certains auteurs ont décrit une transformation maligne possible de ces lésions en lymphome hodgkinien de type $B$ (16).

L'évolution est en règle générale bénigne, justifiant donc une prise en charge le plus souvent conservatrice (2).

Pour les malades HIV positifs, plusieurs auteurs ont démontré une régression complète de ces lésions sous trithérapie anti-rétrovirale. En cas de gêne fonctionnelle, les cytoponctions itératives permettent en général de drainer le contenu du kyste et d'améliorer la symptomatologie (16). Pour plusieurs auteurs, la sclérothérapie semble être un moyen thérapeutique des kystes lymphoépithéliaux (15). Dans notre série, les deux malades ont eu une parotidectomie partielle. L'évolution était bonne avec un recul moyen de 4 ans.

\section{* Le kyste hydatique :}

Les localisations cervico-faciales du kyste hydatique sont rares particulièrement au niveau de la parotide (17).

Le diagnostic peut être évoqué chez un patient en contact avec des chiens, ayant des antécédents de kyste hydatique ou devant l'existence d'une localisation associée (18). Le traitement est chirurgical avec le risque de dissémination de la parasitose en cas de rupture accidentelle du kyste en per-opératoire. II est important de protéger le reste du champ opératoire par des champs imbibés de scolicide (sérum hypertonique, solution de formol, eau oxygénée). Le traitement médical, par le mébendazole (Vermox®) ou l'albendazole (Zentel®), en post opératoire vise la stérilisation des foyers n'ayant pu être réséqués par la chirurgie, cela afin de diminuer le risque de récidive (17).

Dans notre série, le kyste hydatique de la parotide a été retrouvé chez un garçon âgé de 11 ans, qui avait consulté pour une tuméfaction rénitente sous-lobulaire. L'échographie avait confirmé la nature kystique de la masse sans pouvoir cependant en définir l'origine hydatique. L'enfant a eu une parotidectomie partielle emportant le kyste. L'étude histologique a confirmé le diagnostic de localisation hydatique parotidienne. Aucune autre localisation concomitante de kyste hydatique n'a été retrouvée (radiographie du thorax et échographie abdominale normales). L'évolution était bonne avec un recul de 2 ans.

B) Les tumeurs malignes :

Les tumeurs malignes des glandes salivaires sont extrêmement rares avec une prévalence de 1 à 2,5/ 100.000 habitants (1). Elles sont encore plus rares chez l'enfant et représente $5 \%$ de toutes les tumeurs des glandes salivaires de l'enfant (2).

Les tumeurs malignes de la parotide sont dominées par les carcinomes mucoépidermoïdes et le rabdomyosarcome (19).

\section{Le carcinome mucoépidermoïde :}

Le carcinome mucoépidermoïde est la tumeur épithéliale maligne la plus fréquente de l'enfant (20).

L'âge moyen de survenue se situe autour de la 10 ème année. II est rare avant 5 ans (19).

Dans notre série, nous rapportons un cas de carcinome mucoépidermoïde chez 1 garçon âgé de 9 ans qui nous a consulté pour une tuméfaction de la loge parotidienne gauche évoluant depuis 1 an. A l'examen, il avait une tuméfaction sous-lobulaire gauche ferme sans déficit facial. Sur le plan histologique, selon le degré de différentiation 
cellulaire, l'extension de la composante kystique et l'invasion du nerf facial, on distingue les formes de bas grade de malignité, de malignité intermédiaire et de haut grade de malignité.

Chez l'enfant, le carcinome mucoépidermoïde de haut grade de malignité est exceptionnel (21).

Le traitement des carcinomes mucoépidemoïdes est chirurgical. La parotidectomie exofaciale peut être indiquée lorsque la tumeur occupe le lobe superficiel de la parotide. La parotidectomie totale conservatrice du nerf facial est indiquée lorsque le lobe profond de la parotide est atteint ou lorsqu'il y a une atteinte de ganglions intra-parotidiens. L'incidence des métastases ganglionnaires des carcinomes de la parotide étant de 18 à $28 \%$, l'indication de curage ganglionnaire est controversée.

Pour certains auteurs, il est indiqué lorsqu'il existe des adénopathies métastatiques cliniques ou radiologiques $(2,19)$. Pour d'autres, le curage est indiqué pour les tumeurs de taille supérieure à $3 \mathrm{~cm}$, les tumeurs de haut grade de malignité et en cas de paralysie faciale associée (20, 21, 22). Plusieurs études ont montré l'efficacité de la radiothérapie post-opératoire dans le traitement des tumeurs malignes des glandes salivaires tout âge confondu. Chez l'enfant, vu les séquelles possibles de la radiothérapie, celle ci n'est indiquée que pour les tumeurs agressives (invasion péri- neurale, atteinte ganglionnaire), de haut grade de malignité ou en cas de tumeur résiduelle non résécable (21).

Rahbar (21) rapporte 7 cas de carcinomes mucoépidemoïdes chez des enfants dont l'âge variait de 3 à 15 ans. Tous ont été traités chirurgicalement ( 3 parotidectomies totales et 4 parotidectomies partielles). Un curage susomohyoïdien a été réalisé chez un enfant. La radiothérapie a été indiquée pour un malade qui a présenté une récidive locale 12 mois après parotidectomie totale.

Dans notre série, une parotidectomie totale avec un curage sous digastrique, sous maxillaire et sus omo-hyoïdien ont été réalisés. L'examen anatomopathologique avait conclu à un carcinome de bas grade avec absence d'envahissement ganglionnaire ( $\mathrm{N}-$ ). L'évolution était bonne avec absence de récidive. Le recul actuel est de 8 mois.

\section{CONCLUSION}

La glande parotide est le siège le plus fréquent des tumeurs salivaires. Bien que les tuméfactions parotidiennes soient rares chez l'enfant, elles représentent une grande variété de diagnostics histologiques y compris des tumeurs malignes.

\section{REFERENCES}

1. Orvidas LJ, Kasperbauer JL, Lewis JL, Osslen KD, Lesnick TG. Pediatric Parotid Masses. Arch Otolaryngol Head Neck Surg. 2000; 126: 177-84. 2. Ethunandan M, Ethunandan A, Macpherson D, Conroy B, Pratt C. Parotid neoplasms in children: experience of diagnosis and management in a district general hospital. Int J Oral Maxillofac Surg. 2003; 32: 373-7.

3. Abdelkéfi M, Mani R, Ben Ali M, Harrathi K, Mokni M, Belcadhi M et al. Adénome pléomorphe de la parotide: parotidectomie totale ou partielle. J Tun ORL 2003; 11: 43-5.

4. Garcia-Perla A, Munoz-Ramos M, Infante-Cossio P, Mayorga-Jimenez F, Gutierrez -Perez JL, Garcia-Perla A . Pleomorphic adenoma of the parotid in childhood. J Craniomaxillofac Surg 2002; 30: 242-5.

5. Rodriguez KH, Vargas S, Robson C, Perez-Atayde A, Shamberger R, McGill $\mathrm{T} \mathrm{J}$ et al. Pleomorphic adenoma of the parotid gland in children. Int $\mathrm{J}$ Pediatr Otorhinolaryngol 2007; 71: 1717-23.

6. Lin CC, Tsai MH, Huang CC, Hua CH, Tseng HC, Huang ST. Parotid tumors: a 10-year experience. Am J Otolaryngol Head Neck Med Surg 2008; 29: 94100.

7. Sakai E, Yoda T, Shimamoto H, Hirano Y, Kusama M, Enomoto S. Pathologic and imaging findings of an oncocytoma in the deep lobe of the left parotid gland. Int J Oral Maxillofac Surg 2003; 32: 563-5.

8. Bonfils P, Frydman E, Meatchi T, Mimoun M, Preobrajenski ND. Oncocytome massétérin d'origine parotidienne. Rev Stomatol Chir Maxillofac 2007;108:21821

9. Politi M, Toro C, Cian R, Robiony M, Zerman N. Multifocal adenomatous oncocytic hyperplasia of the parotid gland. Oral Oncology 2005; 41:183-7.

10. Mantravadi J, Roth LM, Kafrawy AH. Vascular neoplasms of the parotid gland. Oral Surg Oral. Med Oral Pathol 1993;75:70-5.

11. Buckmiller LM, Francis CL, Glade RS. Intralesional steroid injection for proliferative parotid hemangiomas. Int J Pediatr Otorhinolaryngol 2008; 72:81-7.

12. Mehta RP, Deschler DG. Intraoperative diagnosis of facial nerve schwannoma at parotidectomy. Am J Otolaryngol Head Neck Med Surg 2008;29:126-9.
13. Ulku $\mathrm{CH}$, Uyar $\mathrm{Y}$, Acar $\mathrm{O}$, Yaman $\mathrm{H}$, Avunduk MC. Facial Nerve Schwannomas: A Report of Four Cases and a Review of the Literature. Am J Otolaryngol 2004; 25(6): 426-31.

14. Caughey RJ, May M, Schaitkin BM. Intraparotid facial nerve schwannoma: Diagnosis and Management. Otolaryngol Head Neck Surg 2004;130:586-92.

15. Suskind DL, Tavill MA, Doxycycline SD. Sclerotherapy of benign lymphoepithelial cysts of the parotid: a minimally invasive treatment. Int $\mathrm{J}$ Pediatr Otorhinolaryngol 2000;52: 157-61.

16. Terry JH, Loree TR, Thomas MD, Marti JR. Major Salivary Gland Lymphoepithelial Lesions and the Acquired Immunodeficiency Syndrome. Am J Surg 1991;162:324-9.

17. Karahatay S, Akcam T, Kocaoglu M, Tosun F, Gunhan O. A rare cause of parotid swelling: Primary hydatid cyst. Auris Nasus Larynx 2006;33: 227-29.

18. Safioleas M, Giannopoulos A, Manti C, Stamatakos M, Safioleas K, Stavrou E. Hydatid disease of the parotid gland: A rare case report. Parasitol Int 2007;56: 247-9.

19. Boahene DKO, Olsen KD, Lewis JE, Pinheiro AD, Pankratz VS, Bagniewski SM. Mucoepidermoid Carcinoma of the Parotid Gland. Arch Otolaryngol Head Neck Surg. 2004;130:849-56.

20. Khadaroo BRG, Walton JM, Ramsay JA, Hicks MJ, Archibald SD. Mucoepidermoid Carcinoma of the Parotid Gland: A Rare Presentation in a Young Child. J Pediatr Surg 1998;33(6): 893-5.

21. Rahbar R, Grimmer JF, Vargas SO, Robson CD, Mack Antonio JW, PerezAtayde $R$ et al. Mucoepidermoid Carcinoma of the Parotid Gland in Children A 10-Year Experience. Arch Otolaryngol Head Neck Surg. 2006;132:375-80. 22. Claros P, Dominte G, Claros AJ, Castillo M, Cardesa A, Claros A. Parotid gland mucoepidermoid carcinoma in a 4-year-old child. Int J Pediatr Otorhinolaryngol 2002;63:67-72. 